( 1991 Kluwer Academic Publishers. Printed in the Netherlands.

\title{
A Gauge Field Governing Parallel Transport Along Mixed States
}

\author{
ARMIN UHLMANN \\ Department of Phistcs, Untersity of Letpzig, 701 Letpzig. Germany \\ (Received 3 July 1990)
}

Abstract. At first, a short account is given of some basic notations and results on parallel transport along mixed states. A new connection form (gauge field) is introduced to give a geometric meaning to the concept of parallelity in the theory of density operators.

AMS subject classifications (1980). 81C99, 81D99.

\section{Purification}

It is the aim of this Letter to describe a certain gauge field which is intimately connected with a known [1] natural parallel transport along mixed states. This parallel transport (see below) defines an holonomy and poses the problem of 'topological phases' in analogy to the construction of the Berry phase for curves of pure states $[2,3]$, and for paths of projection operators ('degenerated ground states') [4]. While the construction of the connection form (i.e. the announced gauge field) may be of interest, I start with some arguments in favour of the physical relevance and origin of the parallel transport governed by it.

Let $\mathscr{H}$ be an Hilbert space. A density operator $\varrho$ is a positive trace-class operator with trace one. It describes a state with expectation values

$$
\varrho: A \mapsto \varrho(A):=\operatorname{tr} A \varrho .
$$

The principles of quantum theory allow for the existence of a system, $\mathscr{H}^{\text {ext }}$, larger than that given by $\mathscr{H}$ within which every density operator $\varrho$ can be lifted to a pure vector state $\mid \psi)\left(\psi \mid\right.$ with normalized $\psi \in \mathscr{H}^{\mathrm{ert}}$. The vector state is called a purification of $\varrho$, and $\varrho$ is the reduction of the vector state to $\mathscr{H}$. The purifying lifting is by no means unique: if there is one, then there are many vector states of $\mathscr{H}^{\text {ext }}$ giving $\varrho$ by their reduction to $\mathscr{H}$.

To achieve purification, it is sufficient to consider Hilbert spaces of the form

$$
\mathscr{H}^{\mathrm{ext}}=\mathscr{H} \otimes \mathscr{H}^{\prime},
$$

where the dimension of $\mathscr{H}^{\prime}$ is not smaller than the dimension of $\mathscr{H}$. Reduction to $\mathscr{H}$ means, of course, performing the partial trace with respect of $\mathscr{H}^{\prime}$. Now let $I^{\text {cxt }}$, $I$, and $I^{\prime}$, be the identity operators of the three Hilbert spaces. Every operator $A$ acting on $\mathscr{H}$ determines an operator $A \otimes I^{\prime}$ of the extended Hilbert space, and the 
normalized vector $\psi \in \mathscr{H}^{\mathrm{c} x \mathrm{t}}$ purifies $\varrho$ if always

$$
\varrho(A)=\left(\psi, A \otimes I^{\prime} \psi\right)
$$

is valid. It is further worthwhile to remember that an operator acting on $\mathscr{H}^{\text {ext }}$ commutes with all operators $A \otimes I^{\prime}$ iff it is of the form $I \otimes B^{\prime}$, where $B^{\prime}$ is acting on $\mathscr{H}^{\prime}$.

\section{Parallelity}

If one tries to purify two density operators, $\varrho_{1}$ and $\varrho_{2}$, simultaneously by two normed vectors, $\psi_{1}$ and $\psi_{2}$ of $\mathscr{H}^{\text {ext }}$, the purification ambiguity can be somehow diminished. Namely, these vectors can be chosen as near as possible to each other, i.e. with maximally possible transition probability $[5,6]$ : already given $\psi_{1}$, there is $\psi_{2}$ with

$$
\left(\psi_{1}, \psi_{2}\right) \geqslant\left|\left(\psi_{1}^{\prime}, \psi_{2}^{\prime}\right)\right|
$$

for every pair of vectors, $\psi_{1}^{\prime}, \psi_{2}^{\prime}$, simultaneously purifying $\varrho_{1}$ and $\varrho_{2}$. I describe this situation by calling the pair $\psi_{1}, \psi_{2}$, 'parallel' as a shorthand for 'as parallel as possible' [1]. Indeed, the nonnegative value $\left(\psi_{1}, \psi_{2}\right)$ may be viewed as the cosine of the angle between the unit vectors $\psi_{1}$ and $\psi_{2}$, and it is the larger the smaller the angle is. Its maximal value is the trace norm of $\sqrt{\varrho_{1}} \sqrt{\varrho_{2}}$.

Remark 1. There is a curious question concerning parallelity. Consider $\psi=\lambda_{1} \psi_{1}+\lambda_{2} \psi_{2}$ with a parallel pair $\psi_{1}, \psi_{2}$. Then the reduced density operator of $\psi$, as given by (3), depends on $\varrho_{1}, \varrho_{2}$ only - and not on the choice of the pair of parallel purifications. Are there physically relevant situations where this 'superposition of density operators' has some meaning? See also [7].

Now I consider a given path

$$
s \mapsto \varrho, \quad \text { with } 0 \leqslant s \leqslant 1
$$

of density operators of $\mathscr{H}$ and ask for purifications

$$
s \mapsto \psi, \in \mathscr{H}^{\mathrm{ext}} .
$$

Choosing two nearby parameters, $s$ and $t$, one has

$$
\left\|\psi,-\psi_{t}\right\|^{2}=2-2 \Re\left(\psi_{,}, \psi_{t}\right)
$$

showing a distance minimum for parallel purifications. However, in the case of a path, this cannot be achieved for all its pairs of vectors. A natural way out is to require parallelity only for 'infinitely neighboured' vectors. Hence, the increase in length should be minimal. Because of

$$
\lim c^{-1}\left\|\psi_{s+c}-\psi_{s}\right\|=\sqrt{\left(\dot{\psi}_{s}, \dot{\psi}_{s}\right)},
$$

where $\dot{\psi}$ is the $s$-derivative of $\psi$, the following definition can be given $[8,11]$ : 
The purification $s \mapsto \psi$, of $s \mapsto \varrho_{s}$ is called 'parallel' or a 'parallel transport' if for every purification $s \mapsto \psi$ ', of the same path $s \mapsto \varrho$, it holds

$$
(\dot{\psi}, \dot{\psi}) \leqslant\left(\dot{\psi}^{\prime}, \dot{\psi}^{\prime}\right) \text { for all } s .
$$

It is plain to derive a necessary condition for parallel purifications. Assuming (6) is parallel, one selects self-adjoint operators $B=I \otimes B^{\prime}$ and considers $U(s)=$ $\exp (i s B)$. Then the path $\psi_{i}^{\prime}=U(s) \psi$, is another purification of (5). Inserting this into (9), one gets

$$
0 \leqslant(B \psi, B \psi)+i[(\dot{\psi}, B \psi)-(\psi, B \dot{\psi})] .
$$

This inequality can be valid for all allowed $B$ only if

$$
(\dot{\psi}, B \psi)=(\psi, B \dot{\psi}) \text { for all } B=I \otimes B^{\prime} .
$$

If this equation is valid for all bounded self-adjoint operators $B^{\prime}$ defined on $\mathscr{H}^{\prime}$, then it is true for all bounded operators acting on $\mathscr{H}^{\prime}$. Since the condition (10) is linear in $B$ and, hence, in $B^{\prime}$, and since the linear span of the bounded Hermitian operators contains all bounded operators, (10) holds for all bounded operators.

Before going further, I have to add some statements. (See [11] for their imbedding within a more general context.) (a) Condition (10) is not only necessary but also sufficient - modulo particular regularity requirements. In particular, the rank of the density operators involved should remain constant. If $\mathscr{H}$ is of infinite dimension, the rank of an operator is the dimension of its carrier in the sense of Murray and von Neumann. (b) Under the assumption of statement (a), two parallel purifications of the same path (5) of density operators are isometrically isomorphic, and the partial isometry is of the form $I \otimes B^{\prime}$. (c) In order that (10) give the minimum in (9), the purifying path is assumed to be of norm one. However, sometimes it is convenient to allow arbitrary normalization. This can be done by minimizing not $(\dot{\psi}, \dot{\psi})$ but

$$
\frac{(\dot{\psi}, \dot{\psi})}{(\psi, \psi)}
$$

Only a curve of vectors with constant norm minimizes this expression. (d) A counterpart to this is the following: Let $\psi$, denote a path of vectors with arbitrary (but not vanishing) norms. It fulfills conditions (10) if and only if they are fulfilled by the normalized path $\|\psi\|^{-1} \cdot \psi_{3}$.

\section{The Standard Choice of $\mathscr{H}^{\text {ext }}$}

The standard choice of $\mathscr{H}^{\text {ext }}$ is obtained by choosing the second factor in $\mathscr{H} \otimes \mathscr{H}^{\prime}$ to be the dual of the Hilbert space $\mathscr{H}$. Then the extended Hilbert space becomes the space of the Hilbert-Schmidt operators, $W$, over $\mathscr{H}$. We denote this Hilbert space by $\mathscr{H}^{\prime}$. Its scalar product is well known:

$$
\left(W_{1}, W_{2}\right)=\operatorname{tr} W_{1}^{*} W_{2} .
$$


Here, and from now on, the elements of $\mathscr{W}^{\circ}=\mathscr{H}^{\text {ext }}$ are denoted by $W, W_{1}, \ldots$ and not by the Greek $\psi, \psi_{1}, \ldots$ as before. This should help to distinguish the standard case from more general ones. If $A$ is an operator acting on $\mathscr{H}$, then one has the simple notations

$$
\left(A \otimes I^{\prime}\right) W=A W \quad \text { and } \quad\left(I \otimes A^{\prime}\right) W=W A
$$

(where one can identify $A^{\prime}$ with the dual of $A^{*}$ acting on the dual of $\mathscr{H}$ ). As an application, I rewrite Equation (10) with the help of (12) and (13) to get - replacing $\psi$ by $W-$

$$
\operatorname{tr}\left(\dot{W}^{*} W B-W^{*} \dot{W} B\right)=0
$$

for all $B$. This is nothing but [1]

$$
W^{*} \dot{W}-\dot{W}^{*} W=0 .
$$

In this form, conditions (10) are easier to handle.

Remark 2. There is a similar problem, as in Remark 1, but this time with holonomy. Assume (5) is describing a closed curve of density operators and choose

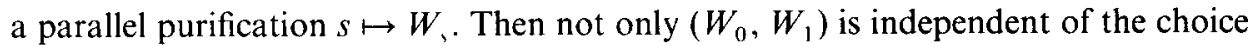
of the parallel purification but also $W W^{*} / \operatorname{tr} W W^{*}$ with $W=W_{0}+W_{1}$. Again, there is the question of whether one can experimentally find such interferences $[1,9,10]$.

\section{Parallelity and a Connection Form}

If 'is naturally a manifold on which one may treat exterior differential forms and further structures. In order to bypass complicated technicalities, I restrict mayself to finite-dimensional Hilbert spaces - although this assumption does not show up explicitly and is probably inessential. We look at $\mathscr{W}^{\prime}$ as a bundle structure the base space of which is the manifold $\Omega$ (with boundary) of all density operators. The projection of this bundle is given by

$$
\pi: W \mapsto \varrho:=W W^{*} /(W, W),
$$

where the point $W=0$ is excluded. If a path in $\Omega$ is lifted to $\mathscr{W}$, the lift is called parallel if (14) holds. The group of unitaries and the multiplicative group of positive reals, acts on a fibre $\pi^{-1} \varrho$. We call

$$
W \mapsto W U
$$

with unitary and $W$-dependent $U$, a (local) gauge transformation and

$$
W \mapsto \lambda W
$$

with $W$-dependent positive real $\lambda$, a scale transformation. At the boundary of $\Omega$, one has to extend (16) by partial isometries of appropriate rank, and the gauge 
group of unitaries has to be replaced by the groupoid of partial isometries - an interesting structure by itself.

In a first step, in examining the parallelity condition, an operator 1-form $\mathbf{G}$ will be defined on $\mathscr{W}$. Its significance is as follows. With $W=W$, parallel, and $\mathbf{G}=G \mathrm{~d} s$, where $G$ is the restriction of the 1 -form to that path, one should have $\dot{W}=G W$. In other words, all parallel curves $W=W$, should be (the) integral curves of the differential form

$$
\vartheta:=\mathrm{d} W-\mathbf{G} W .
$$

Substituting $\dot{W}=G W$ into Equation (14) results in $W^{*}\left(G-G^{*}\right) W=0$. The last equation is fulfilled if

$$
\mathbf{G}=\mathbf{G}^{*}
$$

is satisfied. Considering now the identity

$$
\mathrm{d}\left(W W^{*}\right)=\mathbf{G} W W^{*}+W W^{*} \mathbf{G}+W^{*} \vartheta+\vartheta^{*} W,
$$

$\vartheta$ should vanish along parallel paths. Therefore, one demands

$$
\mathrm{d}\left(W W^{*}\right)=\mathbf{G} W W^{*}+W W^{*} \mathbf{G} .
$$

It is not difficult to see that $\mathbf{G}$ is Hermitian and uniquely determined by Equation (20) if $W$ is invertible. Otherwise, one has to add the subsidiary condition

$$
\langle\phi, \mathbf{G} \phi\rangle=0 \text { for all } \phi \in \mathscr{H} \text {, with } W^{*} \phi=0,
$$

to achieve uniqueness. However, it is not always possible to fulfill this condition: $\mathbf{G}$ may remain undefined at directions $\dot{W}$, where $W$ changes its rank.

Exploiting uniqueness one finds that $\mathbf{G}$ is a gauge invariant form which transforms under rescaling (17) as $\mathbf{G} \mapsto \mathbf{G}-\lambda^{-1} \mathrm{~d} \lambda$.

If $W=W$, is parallel and normalized, $\langle W, W\rangle=1$, then the restriction $G \mathrm{~d} s$ of G along this path depends on $\varrho=\pi(W)$ only. On the unlifted path $s \mapsto \varrho_{s}$, Equation (20) is equivalent to $\varrho=G \varrho+\varrho G$. In this form, Equation (20) has been considered in [9-11].

$\mathbf{G}$ is clearly a gauge invariant 1 -form. Now, I introduce another $\mathbf{l - f o r m}, \mathbf{A}$, which is a connection form for gauge transformations (16). This is done by the following ansatz

$$
W^{*} \mathrm{~d} W-\left(\mathrm{d} W^{*}\right) W=W^{*} W \cdot \mathbf{A}+\mathbf{A} \cdot W^{*} W .
$$

For invertible $W$ all eigenvalues of $W^{*} W$ are positive, and there is a unique solution A. Using this uniqueness, it is a matter of straightforward calculation to see that

$$
\mathbf{A}+\mathbf{A}^{*}=0
$$

and

$$
\mathbf{A} \mapsto \tilde{\mathbf{A}}=: U^{*} \mathbf{A} U+U^{*} \mathrm{~d} U
$$


for every regauging (16). Similar arguments show A to be locally scaleinvariant.

Remaining at the open and dense submanifold of invertible $W$, equation (23) may be used to show the transformation property

$$
\mathrm{d} W \rightarrow W \mathbf{A} \mapsto(\mathrm{d} W-W \mathbf{A}) U
$$

under (16). Clearly, this is the covariant A-derivative of $W$.

There is a further discrete symmetry: Changing $W$ into $\left(W^{*}\right)^{-1} \mathbf{A}$ will not change at all but will reverse the sign of $\mathbf{G}$.

The next assertion is

$$
\mathrm{d} W-W \mathbf{A}-\mathbf{G} W=0 \quad \text { or } \quad \vartheta=W \mathbf{A} .
$$

To see this, one considers (24) for the time being as a defining equation for $\mathbf{G}$. Eleminating $\mathrm{d} W$ in (21) gives the Hermiticity of $\mathbf{G}$. Now inserting $\mathrm{d} W$ into $\mathrm{d}\left(W W^{*}\right)$ gives (20) because of (22). Hence, $\mathbf{G}$ as defined by (24) coincides on the set of nonsingular $W$ with $\mathbf{G}$ as introduced by (19) and (20).

To get the uniqueness of the solution $\mathbf{A}$ of equation (21) for singular $W$, one has to add the condition

$$
\langle\phi, \mathbf{A} \phi\rangle=0 \text { for all } \phi \in \mathscr{H} \text {, with } W \phi=0 .
$$

Then (22) remains true. However, the subsidiary condition cannot be fulfilled at certain tangential directions, and the solution of (21) becomes undefined (singular) on them. This concerns directions where the rank of $W$ is changing.

There is a further point of interest: In extending (23) to the singular case, the gauge transformations (16) have to be enlarged to include partial isometries.

Having a connection form (a gauge field) one is tempted to introduce its curvature form

$$
\mathbf{F}=\mathrm{d} \mathbf{A}+\mathbf{A} \wedge \mathbf{A} .
$$

Performing the exterior derivative of (24), one arrives at

$$
\begin{aligned}
& W(\mathrm{~d} \mathbf{A}+\mathbf{A} \wedge \mathbf{A})+(\mathrm{d} \mathbf{G}-\mathbf{G} \wedge \mathbf{G}) W=0, \\
& (\mathrm{~d} \mathbf{A}+\mathbf{A} \wedge \mathbf{A}) W^{*}=W^{*}(\mathrm{~d} \mathbf{G}+\mathbf{G} \wedge \mathbf{G})
\end{aligned}
$$

\section{Parallel Lifts of Hamiltonian Motions}

My aim is to examine parallel lifts of solutions $t \mapsto \varrho_{t}$ of an evolution equation

$$
i \underline{\varrho}=[H, \varrho], \quad H=H^{*},
$$

with (eventually time-dependent) Hamiltonian $H$. For simplicity, $\varrho$ is assumed to be faithful.

The restriction $G \mathrm{~d} t$ of $\mathbf{G}$ onto this solution fulfills

$$
G=i[\varrho, Y] \text { with } H=\varrho Y+Y \varrho .
$$


Parallel liftings $t \mapsto W$, satisfy the Schrödinger equation

$$
i \dot{W}=H W-W \tilde{H}, \quad \tilde{H}=\tilde{H}^{*} .
$$

This is a special case of the general form of a Schrödinger equation on the Hilbert space $\mathscr{H}$, and the point of the assertion is the Hermiticity of $\tilde{H}$.

For the proof, one needs $\dot{W}=G W$ along parallel liftings to obtain $\tilde{H}=$ $W^{-1}(H-i G) W$. From (28), one infers $H-i G=2 \varrho Y$, and from the polar decomposition

$$
W=\varrho^{12} V
$$

one finally gets

$$
\tilde{H}=2 V^{*} \varrho^{1,2} Y \varrho^{1 / 2} V=2 W^{*} Y W .
$$

Now $\tilde{H}$ appears as a Hamiltonian in the dual of $\mathscr{H}$. Indeed, not only (27) follows from (29) but also

$$
i \frac{\partial}{\partial t} \tilde{\varrho}=[\tilde{H}, \varrho] \quad \text { with } \tilde{\varrho}=W^{*} W .
$$

Finally, inserting (29) into the parallelity condition (14), results in

$$
2 W^{*} H W=W^{*} W \tilde{H}+\tilde{H} W^{*} W .
$$

The two Hamiltonians play a further role: $\mathscr{W}$ is (trivially) a complex analytic manifold. Hence, there is a type decomposition

$$
\mathbf{A}=\mathbf{A}^{(1.0)}+\mathbf{A}^{(0,1)}, \quad \mathbf{G}=\mathbf{G}^{(1,0)}+\mathbf{G}^{(0,1)}
$$

of the 1-forms with respect to this complex analytic structure. Restricting these forms onto a parallel lift (29), $\left(\mathbf{A}^{(0.1)}\right)_{\text {path }}=A^{(0,1)} \mathrm{d} t, \ldots$ results in

$$
A^{(0,1)}=-A^{(1.0)}=i \tilde{H}
$$

and

$$
G^{(0.1)}=-i H, \quad G^{(1.0)}=G+i H .
$$

There are, of course, many further relations if (29) describes a parallel lift of a Hamiltonian evolution for instance, the interconnection with the 'dual' process of lifting $\tilde{\varrho}$.

In the particular case of a time-independent Hamiltonian $H$, one may proceed somewhat more explicitly: (27) yields the well known

$$
\varrho_{t}=\mathrm{e}^{-i t H} \varrho_{0} \mathrm{e}^{t h H}
$$

and $(28)$

$$
Y_{t}=\mathrm{e}^{-t t H} Y_{0} \mathrm{e}^{t t H}, \quad G_{t}=\mathrm{e}^{-t H} G_{0} \mathrm{e}^{t t H} .
$$


It is convenient not to directly take the polar decomposition (30) but to try an ansatz

$$
W_{t}=e^{-t H} \varrho_{0}^{1 / 2} U_{t} V_{0}, \text { i.e. } V_{t}=\mathrm{e}^{-t t H} U_{t} V_{0}
$$

for the parallel lift. Then one gets

$$
\begin{aligned}
& i \dot{U}=-2 \varrho_{0}^{1 / 2} Y_{0} \varrho_{0}^{1 / 2} U, \quad U_{0}=\mathrm{id}, \\
& U_{t}=\exp \left(-2 i t \varrho_{0}^{1 / 2} Y_{0} \varrho_{0}^{1 / 2}\right) .
\end{aligned}
$$

Thus, one gets explicit expressions if one is able to solve

$$
H=\varrho^{1 / 2} X \varrho^{-1 / 2}+\varrho^{-1 / 2} X \varrho^{1 / 2} \text { where } X=\varrho^{1 / 2} Y \varrho^{1 / 2}
$$

at $t=0$. For an example, see [1].

According to Remark 2,

$$
W_{0} W_{i}^{*}=\varrho_{0}^{1 / 2} U_{\imath} \varrho_{0}^{1 / 2} \mathrm{e}^{-t t H}
$$

is seen to depend on $H, t$, and the initial density operator $\varrho_{0}$ only.

\section{References}

1. Uhlmann. A., Rep. Math. Phys. 24, 229 (1986).

2. Berry, M. V, Proc. R. Soc. Lond. A392, 45 (1984)

3. Simon, B., Phvs. Rev. Letl. 51, 2167 (1983).

4. Wilczek, F. and Zee, A , Phys. Ret. Lett. 52, 2111 (1984)

5. Bures. D. J. C., Trans Amer. Math. Soc. 135, 119 (1969).

6. Uhlmann, A.. Rep. Math. Phys. 9, 273 (1976);

Araki, H. and Raggio, G. A., Lett. Math. Phys. 6, 237 (1982),

Alberti, P. M. and Uhlmann, A., Lett. Math. Phys. 7, 107 (1983).

7. Dabrowski, L., A superposition principle for mixed states?, SISSA, Trieste, 156/88/FM.

8. Uhlmann, A., Parallel transport and holonomy along density operators, in H. D. Doebner and J. D. Henning (eds), Differential Geometric Methods in Theorettcal Physics. World Scientific, Singapore, 1987, pp. 246-254.

9. Dabrowskı, L. and Jadczyk, A., Quantum statistical holonomy, Trieste PL-50125 (1988).

10 Dabrowskı, L. and Grosse, H., On quantum holonomy for mixed states, Vienna UWThPh-1988-36.

11. Uhlmann, A., Ann. Phys. 47, 63 (1989). 